\title{
Calcifying Odontogenic Cyst of the Oral Cavity: A Clinical Case and Current Updates on the Ethiopathogenesis
}

\author{
Dardo Menditti', Salvatore D’Amato', Luigi Laino', Antonio Mezzogiorno², \\ Vittorio Salvatore Menditti ${ }^{1}$, Mario Guglielmotti ${ }^{1}$, Alfonso Baldi ${ }^{3 *}$ \\ ${ }^{1}$ Multidisciplinary Department of Medical-Surgical and Odontostomatological Specialties, University of Campania \\ "Luigi Vanvitelli", Naples, Italy \\ ${ }^{2}$ Department of Mental Health and Physics, Preventive Medicine, University of Campania “Luigi Vanvitelli”, Naples, Italy \\ ${ }^{3}$ Department of Environmental, Biological and Pharmaceutical Sciences and Technologies, University of Campania \\ "Luigi Vanvitelli", Naples, Italy \\ Email: *alfonsobaldi@tiscali.it
}

How to cite this paper: Menditti, D., D’Amato, S., Laino, L., Mezzogiorno, A., Menditti, V.S., Guglielmotti, M. and Baldi, A. (2020) Calcifying Odontogenic Cyst of the Oral Cavity: A Clinical Case and Current Updates on the Ethiopathogenesis. Open Journal of Stomatology, 10, 250-257. https://doi.org/10.4236/ojst.2020.109024

Received: August 25, 2020

Accepted: September 18, 2020

Published: September 21, 2020

Copyright $\odot 2020$ by author(s) and Scientific Research Publishing Inc. This work is licensed under the Creative Commons Attribution International License (CC BY 4.0).

http://creativecommons.org/licenses/by/4.0/

\begin{abstract}
Background: The calcifying odontogenic cyst (COC) is a rare pathological entity. It falls into a group of lesions with calcifications that present benign and sometime malignant tumor variants. Case Presentation: In the present study, we report on a case of intraosseous/intrasinusal COC with impacted maxillary canine and dentinoid structures odontoma-like. The clinical, radiographical, histopathological, and molecular characteristics of this pathological entity are discussed in relation also to the problems of differential diagnosis, treatment, and prognosis. Conclusion: The true COC is a rare entity in the oral cavity and represents about less than $1 \%$ of all odontogenic lesions. Careful clinical, instrumental and histological analysis must be performed for odontogenic cysts in order to accomplish the correct surgical act and to avoid recurrence.
\end{abstract}

\section{Keywords}

Calcifying Odontogenic Cyst, Gorlin's Cyst, Impacted Canine Tooth, Odontogenic Cysts, Odontogenic Tumors

\section{Introduction}

Calcifying odontogenic cyst (COC) was first described by Gorlin in 1962. COC is thought to arise from the reduced enamel epithelium surrounding the unerupted crown or from the remnants of the dental lamina. It is a cystic lesion which his- 
tologically shows an odontogenic and ameloblastomatous epithelium lining along with ghost cells and focal calcifications and with nests of an eosinophilic material in the capsule, defined dentinoid [1] [2] [3] [4]. Melanin deposits could be found between epithelium cells [5] [6]. The epithelium can present areas of 6 - 8 cells thick over part of its length in sequence with parts very thin and others arranged in groups like a "button" which proliferate into the lumen or into the supporting connective tissue originated from the basal layer [7]. It can occur as intraosseous (central) more commonly, or extraosseous gingival (peripheral, about 20\%), relating to the presence of odontogenic residues in the bone or gingival area [8] [9]. The intraosseous form is the more common. It is often associated with an impacted tooth and can be asymptomatic, while the extraosseous form usually has only gingival swelling. The most affected sites are the anterior region of the maxilla and the mandible with a certain preference for the canine/premolar teeth. The frequency of the affected sites varies according to the various authors [5] [6]. They are generally asymptomatic, and the radiographs show a unilocular or less frequently multilocular radiolucent lesion with focal areas of radiopacity in extraosseous regions, such as in the gingiva, [8] [9]. The male sex adult seems to be the most frequently affected in the anterior maxilla. From a therapeutic point of view, intraosseous $\mathrm{COC}$ is mainly treated by enucleation, with a low rate of recurrence, occurring in $<5 \%$ of cases, while extra-osseous lesion is treated with excision [1] [4] [9]. There are reports of malignant transformation in recurrent cases of COCs, thus, long-term follow-up of such patients should be performed [5] [9].

The WHO in 2005-classification determined that COC was neoplastic, naming as calcifying cystic odontogenic tumors (CCOT) [3] [6]. In the current WHO classification and also in other proposed classifications on odontogenic cysts, COC is included among the odontogenic cysts "developmental/with tumoral variants" and COC and CCOT are considered synonyms [3] [4]. In fact, some COCs show more aggressive behavior than others and this determines a different consideration in the surgical approach [5] based on the dualistic etiological concept of two biological entities of similar origin but with different biological behavior [1] [2] [3] [6] [7] [8].

Nowadays, all these oral calcifying cystic lesions could be considered as a family of pathologic entities which includes heterogeneous groups of oral cystic lesions with benign, locally aggressive or malignant behavior which can be defined benign odontogenic ghost cell lesions (BOGCL) [10] [11] [12]. The true COC is a rare entity in the oral cavity and represents about less than $1 \%$ of all odontogenic lesions. The aim of present study is to report a rare case of COC with ectopic displacement of maxillary tooth in the sinus cavity.

\section{Case Report}

A 14-year-old male patient was admitted to the Oral Surgery Department of the "Luigi Vanvitelli" University, Naples, Italy, complaining of swelling inside the mouth on the right side of the face. Written informed consent was obtained 
from the patient to report the case. Moreover, he presented sensation of nasal obstruction, and reduction of the sense of smell in the right nasal cavity. Clinically, it showed modest facial asymmetry due to painless growth on the right maxillary region. In the corresponding vestibular fornix, a painless, solid mass covered by a normal-looking mucosa was detected. This mass clearly involved the buccal cortex and extended from tooth 11 to 15 with the absence of 13 and retention of 53. The affected area measured about $6 \mathrm{~cm}$ in its largest diameter. Panoramic radiography revealed a well-defined and homogeneous unilocular radiolucent lesion extending from 12 to 26 (Figure 1A). The lesion showed a mixed hypodense-hyperdense imaging in maxillary right sinus extending super-inferiorly from the alveolar ridge up to the floor of orbit, with the presence of calcified areas and inclusion of the ectopic dental germ 13 in a subocular position. The right lateral $\mathrm{X}$-ray confirmed the aspects of the orto-panoramic radiography with greater evidence (Figure 1B). The CT scan revealed on the axial (Figure 1C) and sagittal (Figure 1D) planes a well-defined hypodense area occupying the sinus cavity and extended beyond its dimensions with a cortical swelling. It contained tooth 13 moved to the subocular site with annexed multiple hyperdense calcifications in the right maxillary sinus, with bone swelling without destruction. On the basis of the radiological investigations, our first diagnosis was of follicular cyst of 13 with ectopic endo-sinusal inclusion of 13 with neighboring dentinoid areas.

The bone access was performed using an ossivorous forceps, respecting the cystic wall. A decompression was performed with aspiration of the cystic liquid. The cystic wall was completely enucleated and the pathological tissue was removed (Figures $1(\mathrm{E})-(\mathrm{G})$ ). Finally, also the impacted tooth was removed. The maxillary sinus was irrigated with hydrogen peroxide to ensure rapid hemostasis. The flap was repositioned and sutured. The patient was discharged after a few hours from surgery in good clinical conditions, with antibiotic and anti-inflammatory therapy. At two-year follow-up, the patient had no sensory deficits or signs of injury recurrence, with improvement in facial asymmetry, and natural reconstitution of the previously expanded bone. No functional deficits were observed.

Histopathologic examination revealed a mono-bilayer cubic-cylindrical epithelial substrate that formed the cystic wall and surrounded the cystic lumen (Figure 1H). Numerous GCs with eosinophilic cytoplasm from probable anomalous intracellular cytoplasmic keratinization were found. Several cells were often arranged in groups like "buttons" which pushed into the cystic lumen. In the fibrous capsule macroscopic areas of eosinophilic matrix referable to dysplastic dentin were observed (Figure 1I). These results were suggestive for the diagnosis of COC.

\section{Discussion}

It is reported that COCs originate from the reduced enamel organ, residual of odontogenic epithelium within the tooth follicle, or from the remnants of the 

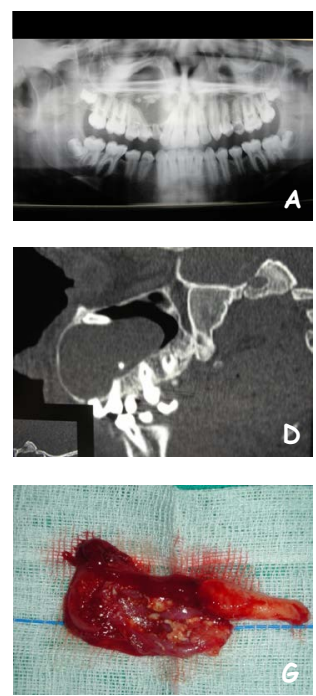
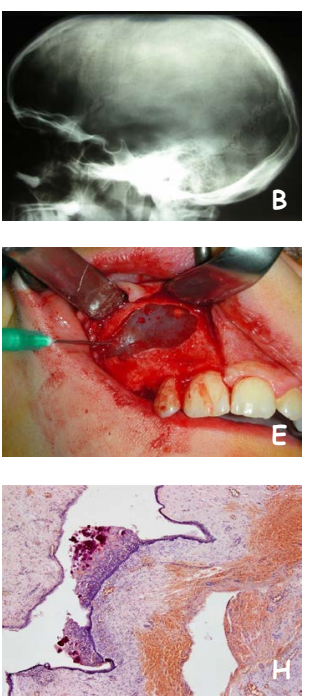
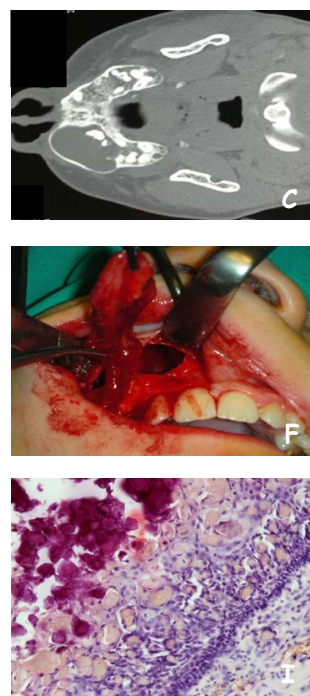

Figure 1. (A) Rx-study of COC that shows the ectopic position of the canine tooth and macro-calcifications of the cyst. (B) Standard lateral Rx-study of the skull: the ectopic tooth is clearly observed. (C) Axial planes CT: the cystic dilatation with anomalous calcifications is well defined. (D) Sagittal planes CT: the ectopic canine and calcification are clarified in the $3 \mathrm{D}$ relationship between them. (E) Intraoperative phases with decompression and enucleation. (F) Intraoperative phases with cystic final enucleation. (G) The cystic specimen after enucleation. $(\mathrm{H})$ Histological aspect of the enucleated cystic lesion. Within a mono-bilayer cubic-cylindrical epithelial substrate that forms the cystic wall and surrounds the cystic lumen, numerous cells are found with eosinophilic cytoplasm from probable anomalous endo-cellular cytoplasmic keratinization, like ghost cells (Hematoxylin and Eosin; original magnification $\times 5$ ). (I) Higher magnification of $(\mathrm{H})$. Numerous cells are often arranged in groups like a "button" which they push into the cystic lumen (Hematoxylin and Eosin; original magnification $\times 10$ ).

odontogenic epithelium, while the peripheral gingival form would derive from rests of the dental lamina ("of Serres") or of the primitive oral epithelium [1] [4] [9]. The mechanisms involved in formation of GC are largely unknown. Most authors consider them an unusual or aberrant form of cellular keratinization, called "ghost cell keratinization" [9]. Odontogenic tissue produces dentin and enamel due to active interactions between odontogenic mesenchyme and epithelium [9] [13]. Therefore, when remnants of the odontogenic epithelium develop without specific differentiation, they could have the potential to produce abnormal calcifications such as enameloid, dentinoid or cementum-like material. COCs lesions, indeed, can be accompanied by odontogenic epithelium, ghost cell changes and odontogenic mesenchyme hyalinization in association with calcified area [1] [2] [6] [9] [12].

An interesting study on the evolution of some fish reports that they secret a substance similar to COC deposits called enameloid, a particular type of highly mineralized dentin rich in amelogenin and type 1 collagen, which would be the result of ectodermal-ectomesenchymal interactions, precisely for the collaboration of ameloblasts and odontoblasts [13]. The phases of accumulation of the calcified material of the COC has not yet been highlighted in pathological tis- 
sues, perhaps because it could be a transitory and short phase and the secreted tissue could change, losing the characteristics of the enameloid-like substance [12] [14].

GCs have been also compared with those found in the calcifying epithelioma of Malherbe in the skin, craniopharyngioma, pilomatrixoma [9] [15]. The GCs are found in groups, particularly in the thicker areas of the epithelial lining [4]. The spinous cells in such situations may be widely separated by intercellular edema and the epithelium around the ghost cells is often convoluted [9].

As histology tells us, the morphology of odontogenic lesions recalls the stages of embryonic development of the teeth [9] [12] while the alterations of the molecular mechanisms of odontogenesis have been associated with the formation of various odontogenic pathological entities [13] [14]. The cells of the ameloblastoma and of COC are very similar for cytology and structure but in the first there is no tendency to calcification [9] [16]. COC, instead, in the advanced phase presents various types of calcifications with dentinoids, calcifications adjacent to the epithelial component, dystrophic mineralization within the GCs [4]. The MAPK, Shh and Wnt signaling pathways are the gene signaling pathways in normal tooth development [12] [17]. Dental hard tissue formation is intimately associated with the Wnt gene signaling pathway in inducing tooth formation [17]. The link between the activation of the pathway Wnt/Beta-catenin, the establishment of GCs and their calcified structures is confirmed by many studies [17] [18]. Genetic alterations affecting abnormal activation of the Wnt pathway have been associated with the pathogenesis of cysts odontogenic tumors in particular BOGCL [12] [18] [19] [20] [21].

It has been reported that the only genetic alteration found in COC is in the beta-catenin, which reinforces the suspicion that this mutation determines an alteration of the Wnt signaling pathway and this would be at the basis of tumorigenesis in COC [22] [23]. Hence, an altered signaling in the odontogenic epithelium coordinated by beta-catenin plays a role in pathogenesis of BOGCL [23] [24] [25].

Beta-catenin mutations with GC formation have been found in ameloblastoma cells confirming a relationship between these two cellular types [26]. It is plausible to think that an abnormal stimulus on cellular remains of the primitive lamina of the Serres determines the development of ameloblastoid cells that have a genetic alteration of the WNT pathway with abnormal beta-catenin activity, which in turn leads to the development of a more differentiated and less aggressive tumor of the ameloblastoma. This hypothesis will explain the similarities of the COC epithelium to that of ameloblastoma and the presence of dysplastic calcification.

\section{Conclusions}

COC is a pathology that may be associated with impacted teeth [27] [28] [29]. The treatment of choice for COC is simple enucleation and curettage. Long-term 
follow-up with radiograms is recommended, since recurrence after 8 years from enucleation have been reported. It also must be underlined that recurrence carries risk of malignant transformation [30] [31]. Therefore, a long follow-up is of crucial importance in the management of this pathological entity.

The presented case recalls all the clinical and histological aspects of a classic COC. It was characterized by the fact that COC completely obliterated the maxillary sinus, bringing the tooth associated with it below the floor of the orbit. The first description of the lesion was radiological: unilocular images well delimited and associated with an impacted and ectopic maxillary canine tooth in a very unusual position, with the divergence of the roots of the teeth involved. The presence of radiopaque structures within the lesion was characteristic; they were of variable numbers and sometimes similar to the teeth. Differential diagnosis must consider all entities of BOGCL and other calcific odontogenic tumors, especially when there is root reabsorption. When the radiopaque structures of the lesion have an irregular radio-density, the differential diagnosis of odontoma, calcifying epithelial odontogenic tumor or ameloblastic fibro-odontoma may be considered. These conditions are managed with more extensive surgery than simple enucleation/curettage.

The enucleation intervention carried out through an oral antrostomy was very intrusive for the considerable size of the lesion and for the adhesions that the capsule and the ectopic tooth had contracted with the antral walls. Enucleation is the choice of treatment for the extraosseous form if the surgeon is certain of the diagnostic COC; in case of doubt at the time of the surgical act, a second surgical moment with curettage can be put in place. A long radiological follow-up must always be done because of the possibility of COC recurrence and especially in case of histological diagnosis other than COC.

\section{Conflicts of Interest}

The authors declare no conflicts of interest regarding the publication of this paper.

\section{References}

[1] Mulvihill, C., Ni Mhaolcatha, S., Brady, P., McKenna, J., Sleeman, D. and Fitzgibbon, J. (2020) Calcifying Odontogenic Cyst: A Case Report. Oral Surgery, 13, 177. https://doi.org/10.1111/ors.12477

[2] El-Naggar, A.K., Chan, J.K.C., Grandis, J.R., Takata, T. and Slootweg, P.J., Eds. (2017) WHO Classification of Head and Neck Tumours. World Health Organization Classification of Tumours. IARC Press, Lyon.

[3] Soluk-Tekkeşin, M. and Wright, J.M. (2018) The World Health Organization Classification of Odontogenic Lesions: A Summary of the Changes of the 2017 (4th) Edition. Turk Patoloji Dergisi, 34.

[4] Menditti, D., Laino, L., Domenico, M.I., Troiano, G., Guglielmotti, M., Sava, S., Mezzogiorno, A. and Baldi, A. (2018) Cysts and Pseudocysts of the Oral Cavity: Revision of the Literature and a New Proposed Classification. In Vivo, 32, 999-1007. https://doi.org/10.21873/invivo.11340 
[5] Santosh, A.B.R. and Ogle, O.E. (2020) Odontogenic Tumors. Dental Clinics of North America, 64, 121-138. https://doi.org/10.1016/j.cden.2019.08.008

[6] Ledesma-Montes, C., Gorlin, R.J., Shear, M., et al. (2008) International Collaborative Study on Ghost Cell Odontogenic Tumors: Calcifying Cystic Odontogenic Tumor, Dentinogenic Ghost Cell Tumor and Ghost Cell Odontogenic Carcinoma. Journal of Oral Pathology \& Medicine, 37, 302-308. https://doi.org/10.1111/j.1600-0714.2007.00623.x

[7] Mokhtari, S., Mohsenifar, Z. and Ghorbanpour, M. (2013) Predictive Factors of Potential Malignant Transformation in Recurrent Calcifying Cystic Odontogenic Tumor: Review of the Literature. Case Reports in Pathology, 2013, Article ID: 853095. https://doi.org/10.1155/2013/853095

[8] Chrcanovic, B.R. and Gomez, R.S. (2016) Peripheral Calcifying Cystic Odontogenic Tumor and Peripheral Dentinogenic Ghost Cell Tumor: An Updated Systematic Review of 117 Cases Reported in the Literature. Acta Odontologica Scandinavica, 74, 591-597. https://doi.org/10.1080/00016357.2016.1236986

[9] Barnes, L., Eveson, J.W., Reichart, P. and Sidransky, D. (Eds) (2005) World Health Organization Classification of Tumours: Pathology and Genetics, Head and Neck Tumours. IARC Press, , Lyon

[10] de Arruda, J., Monteiro, J., Abreu, L.G., et al. (2018) Calcifying Odontogenic Cyst, Dentinogenic Ghost Cell Tumor, and Ghost Cell Odontogenic Carcinoma: A Systematic Review. Journal of Oral Pathology \& Medicine, 47, 721-730. https://doi.org/10.1111/jop.12727

[11] Irani, S., Foroughi, F. (2017) and Histologic Variants of Calcifying Odontogenic Cyst: A Study of 52 Cases. The Journal of Contemporary Dental Practice, 18, 688-694. https://doi.org/10.5005/jp-journals-10024-2108

[12] Rosa, A.C.G., Teixeira, L.N., Passador-Santos, F., et al. (2019) Benign Odontogenic Ghost Cell Lesions Revisited and New Considerations on Dysplastic Dentin. Clinical Oral Investigations, 23, 4335-4343. https://doi.org/10.1007/s00784-019-02863-7

[13] Kawasaki, K., Suzuki, T. and Weiss, K.M. (2005) Phenogenetic Drift in Evolution: the Changing Genetic Basis of Vertebrate Teeth. Proceedings of the National Academy of Sciences of the United States of America, 102, 18063-18068. https://doi.org/10.1073/pnas.0509263102

[14] Diniz, M.G., Gomes, C.C., de Sousa, S.F., Xavier, G.M. and Gomez, R.S. (2017) Oncogenic Signaling Pathways in Benign Odontogenic Cysts and Tumours. Oral Oncology, 72, 165-173. https://doi.org/10.1016/j.oraloncology.2017.07.021

[15] Rumayor, A., Carlos, R., Kirsch, H.M., de Andrade, B.A., Romañach, M.J. and de Almeida, O.P. (2015) Ghost Cells in Pilomatrixoma, Craniopharyngioma, and Calcifying Cystic Odontogenic Tumor: Histological, Immunohistochemical, and Ultrastructural Study. Journal of Oral Pathology \& Medicine, 44, 284-290. https://doi.org/10.1111/jop.12234

[16] Sekine, S., Sato, S., Takata, T., Fukuda, Y., Ishida, T., Kishino, M., et al. (2003) Beta-Catenin Mutations Are Frequent in Calcifying Odontogenic Cysts, But Rare in Ameloblastomas. The American Journal of Pathology, 163, 1707-1712. https://doi.org/10.1016/S0002-9440(10)63528-6

[17] Duan, P. and Bonewald, L.F. (2016) The Role of the Wnt/ß-Catenin Signaling Pathway in Formation and Maintenance of Bone and Teeth. The International Journal of Biochemistry \& Cell Biology, 77, 23-29. https://doi.org/10.1016/j.biocel.2016.05.015

[18] Wang, X.-P., O’Connell, D.J., Lund, J.J., Saadi, I., Kuraguchi, M., Turbe-Doan, A., et 
al. (2009) Apc Inhibition of Wnt Signaling Regulates Supernumerary Tooth Formation during Embryogenesis and Throughout Adulthood. Development, 136, 1939-1949. https://doi.org/10.1242/dev.033803

[19] Wang, J. and Feng, J.Q. (2017) Signaling Pathways Critical for Tooth Root Formation. Journal of Dental Research, 96, 1221-1228. https://doi.org/10.1177/0022034517717478

[20] Kim, S.A., Ahn, S.G., Kim, S.G., Park, J.C., Lee, S.H., Kim, J., et al. (2007) Investigation of the Betacatenin Gene in a Case of Dentinogenic Ghost Cell Tumor. Oral Surgery, Oral Medicine, Oral Pathology, Oral Radiology, and Endodontology, 103, 97-101. https://doi.org/10.1016/j.tripleo.2005.10.037

[21] Ahn, S.G., Kim, S.A., Kim, S.G., Lee, S.H., Kim. J. and Yoon, J.H. (2008) $\beta$-Catenin Gene Alterations in a Variety of So-Called Calcifying Odontogenic Cysts. APMIS, 116, 206-211. https://doi.org/10.1111/j.1600-0463.2008.00893.x

[22] Polakis, P. (2012) Wnt Signaling in Cancer. Cold Spring Harbor Perspectives in Biology, 4, a008052. https://doi.org/10.1101/cshperspect.a008052

[23] de Sousa, S.f., Moreira, R.G., Gomez, R.S. and Gomes, C.C. (2016) Interrogation of Cancer Hot Spot Mutations in Tumor Suppressor and Oncogenic Genes in the Calculation of Cystic Odontogenic Tumor. Oral Oncology, 57, e1-e3. https://doi.org/10.1016/j.oraloncology.2016.04.010

[24] Kim, T.H., Lee, J.Y., Baek, J.A., et al. (2011) Constitutive Stabilization of ß-Catenin in the Dental Mesenchyme Leads to Excessive Dentin and Cementum Formation. Biochemical and Biophysical Research Communications, 412, 549-555. https://doi.org/10.1016/j.bbrc.2011.07.116

[25] Xavier, G.M., Patist, A.L., Healy, C., Pagrut, A., Carreno, G., Sharpe, P.T., et al. (2015) Activated WNT Signaling in Postnatal SOX2-Positive Dental Stem Cells Can Drive Odontoma Formation. Scientific Reports, 5, Article No. 14479. https://doi.org/10.1038/srep14479

[26] Dutra, S., Pires, F., Armada, L. and Azevedo, R. (2017) Immunoexpression of Wnt/ $\beta$-Catenin Signaling Pathway Proteins in Ameloblastoma and Calcifying Cystic Odontogenic Tumor. Journal of Clinical and Experimental Dentistry, 9, e136-140. https://doi.org/10.4317/jced.53100

[27] Yukimori, A., Oikawa, Y., Morita, K.I., et al. (2017) Genetic Basis of Calcifying Cystic Odontogenic Tumors. PLoS ONE, 12, e0180224. https://doi.org/10.1371/journal.pone.0180224

[28] Shiva, A. and Nosrati, K.A. (2015) Calcifying Odontogenic Cyst Associated with Complex Odontoma: A Case Report. Journal of Babol University of Medical Sciences, $17,57-61$.

[29] Mortazavi, H. and Baharvand, M. (2016) Jaw Lesions Associated with Impacted Tooth: A Radiographic Diagnostic Guide. Imaging Science in Dentistry, 46, 147-157. https://doi.org/10.5624/isd.2016.46.3.147

[30] Uzun, T. and Çinpolat, E. (2019) Calcifying Odontogenic Cyst Associated with the Impacted Third Molar: A Case Report. Pan African Medical Journal, 33, 151. https://doi.org/10.11604/pamj.2019.33.151.17601

[31] Li, B.B. and Gao, Y. (2009) Ghost Cell Odontogenic Carcinoma Transformed from a Dentinogenic Ghost Cell Tumor of Maxilla after Multiple Recurrences. Oral Surgery, Oral Medicine, Oral Pathology, and Oral Radiology, 107, 691-695. https://doi.org/10.1016/j.tripleo.2009.01.008 